\title{
Contents
}

\section{Classical Systems}

Atomic models; radiation; Rutherford scattering; specific heats; normal modes of vibration.

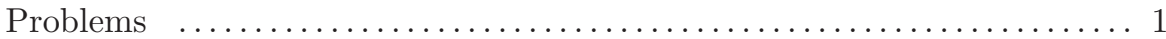

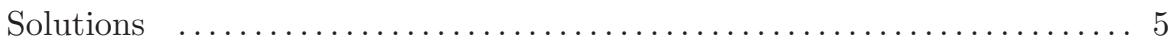

\section{Old Quantum Theory}

Spectroscopy and fundamental constants; Compton effect; Bohr-Sommerfeld quantization; specific heats; de Broglie waves.

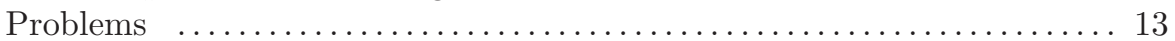

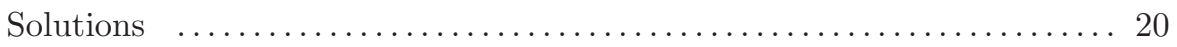

\section{Waves and Corpuscles}

Interference and diffraction with single particles; polarization of photons; Malus' law; uncertainty relations.

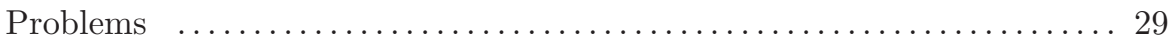

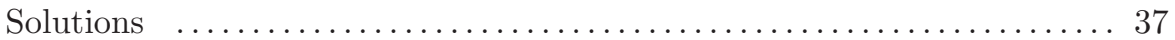

\section{$4 \quad$ States, Measurements and Probabilities}

Superposition principle; observables; statistical mixtures; commutation relations.

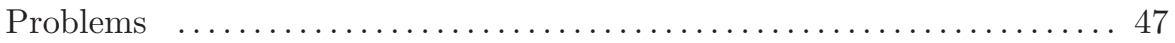

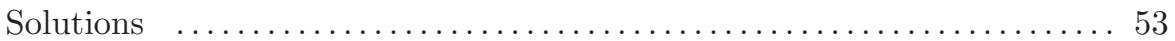

\section{Representations}

Representations; unitary transformations; von Neumann theorem; coherent states; Schrödinger and momentum representations; degeneracy theorem.

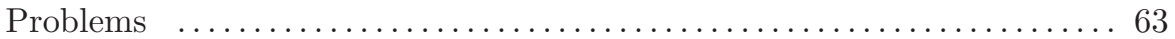

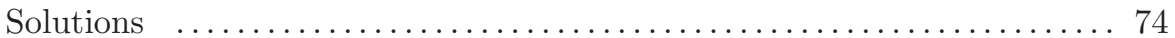

\section{One-Dimensional Systems}

Nondegeneracy theorem; variational method; rectangular potentials; transfer matrix and $S$-matrix; delta potentials; superpotential; completeness.

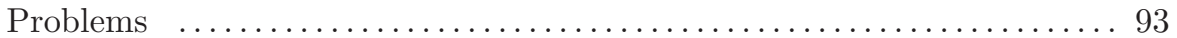

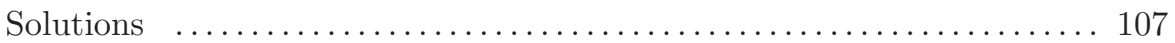




\section{Time Evolution}

Time evolution in the Schrödinger and Heisenberg pictures; classical limit; time reversal; interaction picture; sudden and adiabatic approximations.

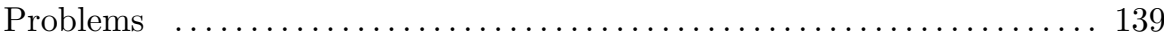

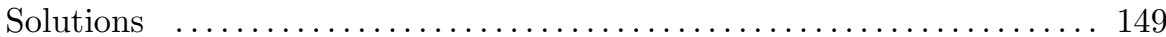

\section{Angular Momentum}

Orbital angular momentum: states with $l=1$ and representations; rotation operators; spherical harmonics; tensors and states with definite angular momentum $(l=1, l=2)$.

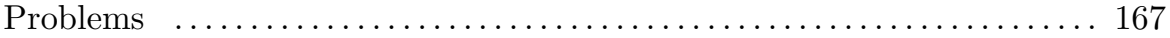

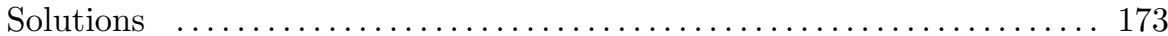

\section{Changes of Frame}

Wigner's theorem; active and passive point of view; reference frame: translated, rotated; in uniform motion; in free fall, rotating.

Problems ............................................... 185

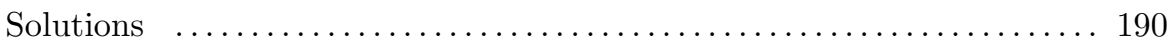

\section{Two and Three-Dimensional Systems}

Separation of variables; degeneracy theorem; group of invariance of the two-dimensional isotropic oscillator.

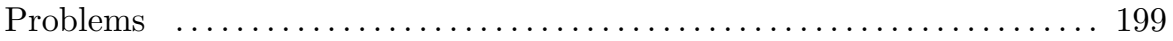

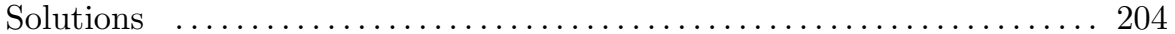

\section{Particle in Central Field}

Schrödinger equation with radial potentials in two and three dimensions; vibrational and rotational energy levels of diatomic molecules.

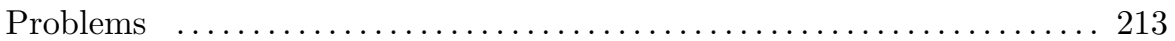

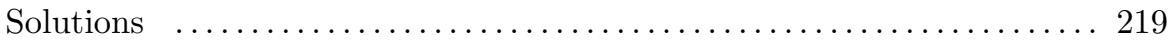

\section{Perturbations to Energy Levels}

Perturbations in one-dimensional systems; Bender-Wu method for the anharmonic oscillator; Feynman-Hellmann and virial theorems; "no-crossing theorem"; external and internal perturbations in hydrogen-like ions.

Problems . . ........................................ 231

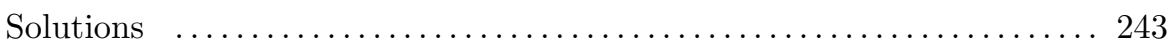

\section{Spin and Magnetic Field}

Spin $\frac{1}{2}$; Stern and Gerlach apparatus; spin rotations; minimal interaction; Landau levels; Aharonov-Bohm effect.

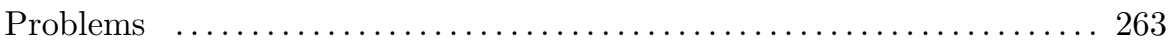

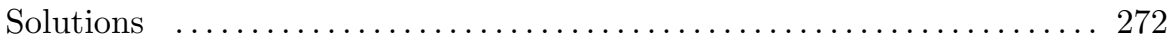

\section{Electromagnetic Transitions}

Coherent and incoherent radiation; photoelectric effect; transitions in dipole approximation; angular distribution and polarization of the emitted radiation; life times.

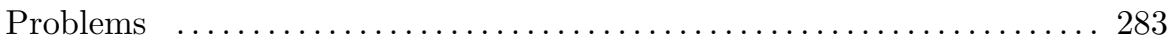

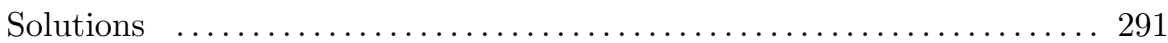




\section{Composite Systems and Identical Particles}

Rotational energy levels of polyatomic molecules; entangled states and density matrices; singlet and triplet states; composition of angular momenta; quantum fluctuations; EPR paradox; quantum teleportation.

Problems . . ......................................... 301

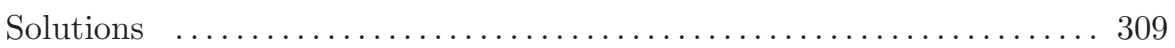

\section{Applications to Atomic Physics}

Perturbations on the fine structure energy levels of the hydrogen atom; electronic configurations and spectral terms; fine structure; Stark and Zeeman effects; intercombination lines.

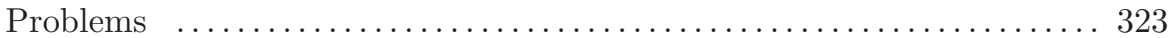

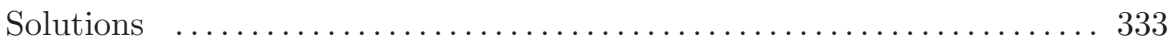

Appendix A Physical Constants $\ldots \ldots \ldots \ldots \ldots \ldots \ldots \ldots \ldots \ldots \ldots$

Appendix B Useful Formulae $\ldots \ldots \ldots \ldots \ldots \ldots \ldots \ldots \ldots \ldots \ldots$

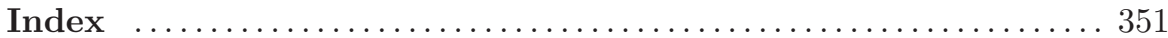

